\title{
Growth Surface Model with Non Active Sites
}

\author{
M. Santos ${ }^{(\star)}$ and W. Figueiredo ${ }^{(\star \star)}$ \\ ${ }^{(\star)}$ Centro de Ensino de São José, Universidade do Vale do Itajaí, 88122-000, São José, SC, Brazil \\ ${ }^{(\star *)}$ Departamento de Física, Universidade Federal de Santa Catarina, 88040-900, Florianópolis, SC, Brazil
}

Received on 5 September, 2003

\begin{abstract}
In this work we studied the effects of non active sites on the substrate of a growing surface. In our model a particle only sticks at the surface if the site where it falls is an active site. However, we allow the deposited particle to diffuse along the surface in accordance with some previously defined mechanism. Using Monte Carlo simulations, and some analytical results, we have investigated the model in (1+1) and (2+1) dimensions, considering different relaxation mechanisms. We show that the inclusion of non active sites is a crucial point in the model. In fact, we have observed that the saturation regime always disappears and that the values of the growth exponent $\beta$ go to one, at large times, for any mechanism of diffusion we considered in one and two dimensions.
\end{abstract}

\section{Introduction}

Growth surface models have been extensively studied in the last years in order to better understand the conditions under which the growing of a surface is affected. The interest in this phenomenon is related to the great variety of technological applications that depend on the morphology of the surface [1-3]. On the other hand, the growth of a surface, despite to be a typical non equilibrium process, presents a scaling behaviour for the fluctuations of the interface width around its mean, which depends on time and length of the sample [4]. Then, much attention has been also devoted to the study of the growing processes, particularly with respect to the universality class in which a particular model can be classified [5-12]. In this work we have studied growth surface models in one and two dimensions considering the existence of defects on the substrate where the particles land. In our model, the particles can not collide with given surface sites. As we will see later, the introduction of defects on the substrate is a crucial factor to determine the morphology of the surface. For the best of our knowledge, the growth surface models found in literature do not consider the possibility of defects on the substrate. Our paper is organized as follow. In section II, we present the Vicsek-Family scaling relation and the models considered in this study. Besides, we also present the analytical results for a simple deposition model in $d+1$ dimensions, as well as we show the results of Monte Carlo simulations for models that take into account some relaxation mechanism in $d+1$ dimensions, with $d=1,2$. Finally, in section III, we draw our conclusions.

\section{Models, scaling laws and results}

The simplest model to describe the growing of a surface is the random deposition model (RD) [1,2]. In this model, a site on the substrate is randomly chosen and its height is increased by one unit. Therefore, a particle immediately sticks at the place where it hits. More realistic models consider an additional step: after the deposition, the particle can move to a neighbor site following a given rule. In the Family model, for instance, the particle migrates to the site with the smallest height among its first neighbor sites [4]. On the other hand, in the large curvature model (LC) $[5,12]$, the particle moves to the nearest-neighbor site with the maximum value of the local curvature. In general, as a result of the particle diffusion, the interface becames smoother. In the models we consider, we used a substrate in $d$ dimensions with linear size $L$ and we took periodic boundary conditions in the direction perpendicular to the growth surface. Besides, at the initial time $t=0$ all the sites of the substrate are empty and the time unit is the conventional Monte Carlo step (MCs) in which $L^{d}$ particles are deposited.

In order to characterize the growth of a surface two functions are defined: (a) the mean value of the height of the surface and the fluctuations of the height of the surface around its mean value. For a substrate of linear size $L$ in $d$ dimensions these quantities are defined, respectively, by

$$
\bar{h}(t)=\frac{1}{L^{d}} \sum_{i} h(i, t)
$$

and

$$
w(L, t)=\left\langle\frac{1}{L^{d}}\left[\sum_{i}(h(i, t)-\bar{h}(t))^{2}\right]^{\frac{1}{2}}\right\rangle .
$$

Here, $h(i, t)$ is the height at the position of the site $i$, the sum extends over the $L^{d}$ sites of the lattice and the symbol $\langle\ldots\rangle$ means an average over the $K$ independent samples. The quantity $w(L, t)$, also known as the interface width, is a measure of the roughness of the surface at time $t$, for a particular value of $L$. The scaling behavior dependent on time $t$ and length $L$ of the growth model can be summarized by the Family-Vicsek scaling law [4]

$$
w(L, t)=t^{\beta} F\left(\frac{L}{t^{1 / z}}\right),
$$


where the scaling function $F(u)$ is constant for $u>>1$ and scales with $u^{\alpha}$ for $u<<1$. Besides, $t^{\star} \propto L^{z}$ represents a characteristic time related to the change of the behavior of the surface roughness. For $t<<t^{\star}$ the roughness of the surface increases in accordance with $w \propto t^{\beta}$, while for $t>>t^{\star}$ the roughness of the surface reaches a saturation regime in which the value of the roughness is constant but dependent on the lattice length $w \propto L^{\alpha} . \alpha, \beta$ and $z$ are the roughness, growth and dynamic exponents, respectively, and they are related by $\beta=\alpha / z$. In the following table we show the values of the growth exponents for the models considered in this work.

\begin{tabular}{|c|c|c|c|}
\hline model & $\alpha$ & $\beta$ & $z$ \\
\hline RD & - & $1 / 2$ & - \\
\hline Family & $(2-d) / 2$ & $(2-d) / 4$ & 2 \\
\hline LC & $(4-d) / 2$ & $(4-d) / 8$ & 4 \\
\hline
\end{tabular}

As we can see, only the exponent $\beta$ is defined for the RD in $d$ dimensions. On the other hand, the exponents $\alpha$ and $\beta$ are zero for the Family model in $d=2$ dimensions and for the LC model in $d=4$. The interface width presents a logarithmic behavior for the Family model in $d=2$ and the LC model in $d=4$.

One of the features that have received little attention in growth models is related to the nature of the substrate. As we mentioned before, generally we have a flat substrate in a regular lattice (square, triangular, hexagonal, etc), in which the sites are initially empty. However, in a few cases, the substrate was considered to be a fractal structure or at the initial time it was not empty. To include defects on the substrate, we have considered a model in which a fixed density of sites are non active, that is, particles landing on the surface can not directly stick at these sites. However, if a relaxation process is possible, the non active sites can be occupied by migration. We will see that the consideration of non active sites over a substrate completely changes the profile of the surface roughness.

Now, we present some results for the growth models taking into account the presense of defects on the substrate. Firstly, we present an exact calculation of the surface roughness for the RD model in $d+1$ dimensions. So, let us consider a $d$-dimensional substrate with $l=L^{d}$ sites, where $0<m<l$ are active sites, that is, sites where particles can collide and stick. We randomly choose an active site and we increase its height by one unit. By doing so, the height of the $l-m$ inactive sites is zero at any time. If the time unit is defined by the deposition of $l$ particles, and if the flow of particles that arrive at the surface is constant, then, after a time $t, N=l t$ particles are deposited on the substrate. In this model, the height at each site of the surface grows independently (the sites are uncorrelated) with probability $p=1 / m$. Therefore, the probability that an active has height $h$ after the deposition of $N$ particles is given by the binomial distribution

$$
P(h, N)=\frac{N !}{h !(N-h) !} p^{h}(1-p)^{N-h} .
$$

The mean value of the height of the surface is $N / l=t$.

In order to find an exact expression for the roughness of the surface we rewrite the equation (2.2) taking into account that only $m$ sites are active

$$
w^{2}(l, t)=\bar{h}^{2}(t)+\frac{1}{l} \sum_{i=1}^{m} h^{2}(i, t)-\frac{2 \bar{h}(t)}{l} \sum_{i=1}^{m} h(i, t) .
$$

But, if $N$ particles are deposited onto the $m$ active sites with same probability, then we can define

$$
<h^{\star}(t)>=\frac{1}{m} \sum_{i=1}^{m} h(i, t)
$$

and

$$
<h^{\star 2}(t)>=\frac{1}{m} \sum_{i=1}^{m} h^{2}(i, t) .
$$

On the other hand, the above mean values can be written as

$$
<h^{\star}(t)>=\sum_{h=1}^{N} h^{\star}(t) P(h, N)
$$

and

$$
<h^{\star 2}(t)>=\sum_{h=1}^{N} h^{\star 2}(t) P(h, N)
$$

Substituting $P(h, N)$ given by eq. (2.4) in the last two equations we obtain, after some algebraic manipulations,

$$
<h^{\star}(t)>=\frac{N}{m}
$$

and

$$
<h^{\star 2}(t)>=N p(1-p)+N^{2} p^{2} .
$$

Besides, as $\bar{h}(t)=t=N / l$ and $p=1 / m$ we can easily show that the equation (2.5) becomes

$$
w^{2}(l, t)=\left(\frac{l-m}{m}\right) t^{2}+\left(\frac{m-1}{m}\right) t
$$

From the above equation, we observe a very interesting behavior of the interface width as a function of the time. As expected, the roughness does not saturate, but at the initial times $\left(t<<t_{c}\right)$ the roughness scales as $w \propto t^{1 / 2}$, while at large times $\left(t>>t_{c}\right)$ the roughness grows as $w \propto t$ for $m>1$, where $t_{c}$ is a characteristic time of the model where the linear and quadratic terms give the same contribution to the roughness of the surface. This result implies that $\beta=1 / 2$ at the initial times and $\beta=1$ at long times. Then, independently of the inactive sites density over the substrate, there is a change in the dynamical behavior of the surface roughness when defects are present. 


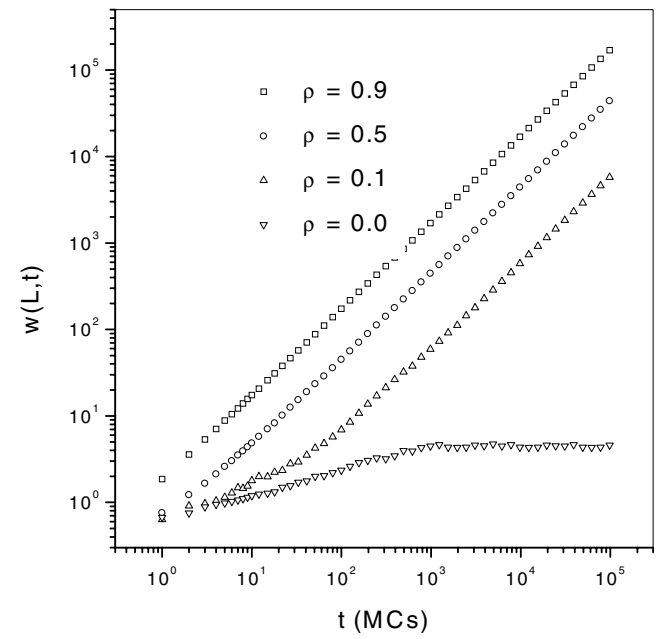

Figure 1. Plot of the interface width versus time for the Family model in $d=1$ and with $L=1000$ for different values of the density of non active sites.

In the RD model with defects a particle can not stick at an inactive site and, as migration is not permitted to these sites, their local heights always remain zero. In principle, this fact could be associated with the anomalous behavior of the roughness as a function of time. Then, in order to get a better understanding of this behavior we have considered models in which inactive sites can be reached by some type of migration mechanism. We first consider the mechanism defined for the Family model in which the particle can move to the nearest-neighbor site with the smallest height after its deposition. We remember that particles only collide with a given site if it is an active one. To calculate the roughness of the surface for this model in $1+1$ and $2+1$ dimensions we have used the Monte Carlo method. In Fig. 1 we show the behavior of the interface width as a function of time for a linear substrate with length $L=1000$, and four different values of the density of inactive sites, $\rho=1-m / L^{d}$. As we can see, the dynamical behavior of the roughness for this model changes completely if $\rho \neq 0$. In fact, the behaviour of this quantity is equivalent to that RD one when defects are present on the substrate. The same occurs for the model in $2+1$ dimensions as shown in Fig. 2. Again, the roughness of the surface does not saturate, and at large times, $w \propto t$ for any value of the linear size of the substrate and density of inactive sites. To draw this figure we have used a quadratic lattice with linear length $L=256$. We also repeated the same calculations as above for the LC model. In this model, the particle landing on an active site $i$ of the substrate migrates to its first neighbor site with the maximum value of the curvature $C(i)=\sum_{j=1}^{n}[h(j)-n h(i)]$, where the sum extends to the first neighbor sites, and $n=2(d=1)$ or $n=4(d=2)$. In Figs. 3 and 4 we show the dynamical behavior of the roughness of the surface for this model as a funtion of the time for one and two dimensional substrates, respectively. Again, the roughness of the surface does not saturate showing an anomalous behavior as seen for the other models considered in this work. The linear size of the lattices used in these figures are $L=1000$ for the linear lattice and $L=256$ for the square lattice.

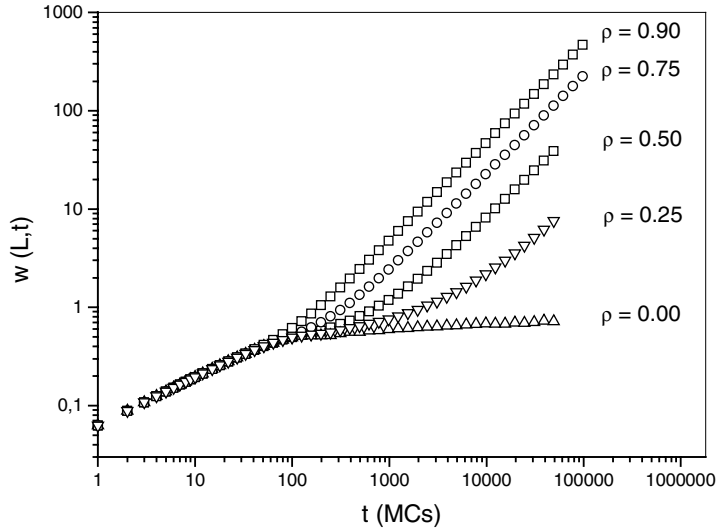

Figure 2. Interface width as a function of time for the Family model in two dimensions for different values of the density of non active sites. We have used $L=256$ sites in this figure.

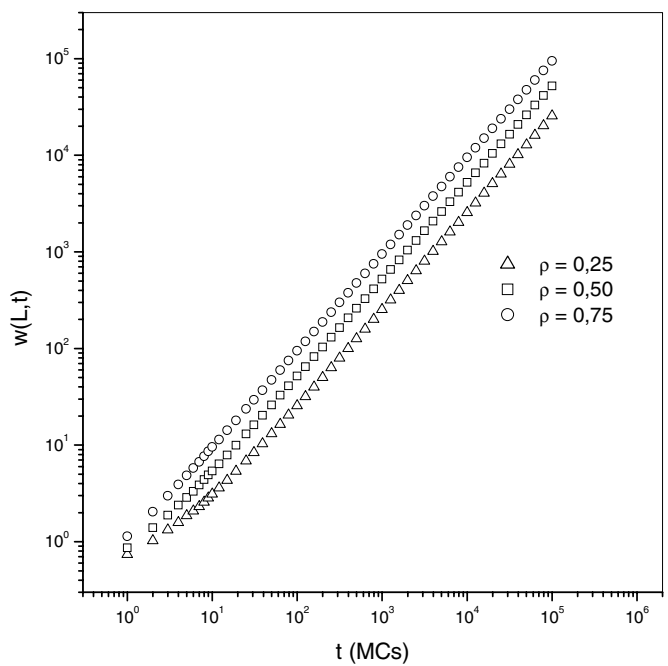

Figure 3. Plot of the interface width versus time for the Large Curvature model in $d=1$ and with $L=1000$ for different values of the non active sites density.

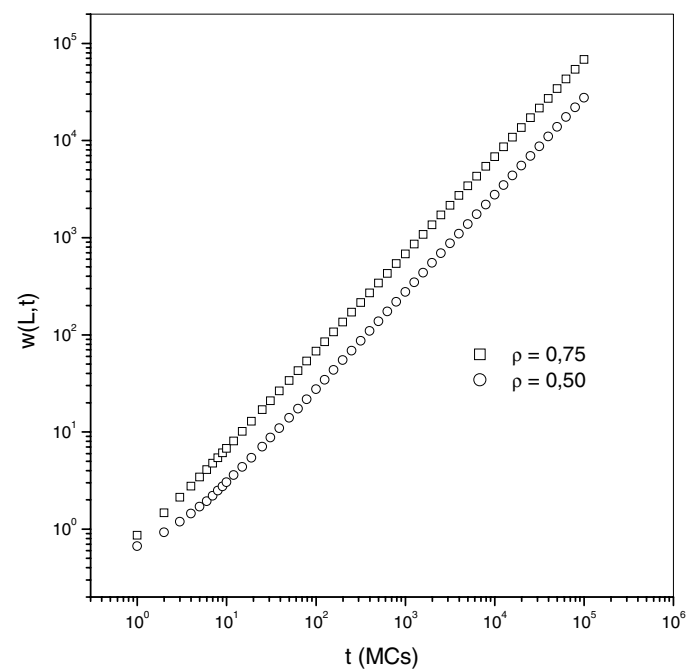

Figure 4. Interface width as a function of time for the Large Curvature model in two dimensions for different values of the density of non active sites. We have used $L=256$ sites in this figure. 


\section{Conclusions}

In this work we have considered the presence of non active sites over the substrate for some growth models. By an exact calculation for the RD model and Monte Carlo simulations for the Family and LC models, for which relaxation mechanisms were considered, we have shown that the inclusion of defects is crucial to determine the morphology of the interface. In fact, we found that the interface width does not saturate even when relaxation processes are present. Besides, the growth exponent $\beta$ characterizing the dependence of the roughness on time also changes. At small times, the value of the exponent $\beta$ is the same as the one of the standard model (no defects present) and, at large times, $\beta=1$ for all the models we considered.

\section{Acknowledgements}

The authors acknowledge the Brazilian agencies FUNCITEC and CNPq by the financial support. They are also grateful to the comments of the referee.

\section{References}

[1] A.-L. Barabasi and H. E. Stanley, Fractal concepts in surface growth, Cambridge University Press, Cambridge (1995).
[2] P. Meakin, Fractals, Scaling, and Growth far from equilibrium, Cambridge University Press, Cambridge (1998).

[3] T. A Witten and L. M. Sander, Phys. Rev. Lett. 47, 1400 (1981).

[4] F. Family and T. Vicsek, J. Phys. A 18, L75 (1985).

[5] S. Das Sarma, C. J. Lanczyncki, R. Kotlyar, and S. Ghaisas, Phys. Rev. E 53, 359 (1996).

[6] J. Krug, Adv. Phys. 46, 139 (1997).

[7] D. P. Landau and S. Pal, Thin Solid Films 272, 184 (1996).

[8] A. P. F. Atman and J. G. Moreira, Eur. Phys. J. B 16, 501 (2000).

[9] A. Chame and F. D. A. Aarão Reis, Phys. Rev. E 66, 051104 (2002).

[10] M. Santos, W. Cavalcanti, A. A. Pasa, and W. Figueiredo, Physica A 308, 313 (2002).

[11] W. Cavalcanti, M. Santos, and W. Figueiredo, Physica A 322, 313 (2003)

[12] J. M. Kim and S. Das Sarma, Phys. Rev. Lett. 72, 2903 (1994). 